\title{
Supersymmetry and Neutrino Masses
}

\author{
by Ernest Ma \\ Physics Department, University of California, Riverside, CA 92521, USA \\ E-mail: ma@ucrpho.ucr.edü
}

ABstRACT: Neutrino masses are zero in the minimal supersymmetric standard model. I discuss how they may become nonzero with new interactions which may or may not violate $R$-parity conservation.

KEYWORDS: 'neutrino mass, supersymmetry, $R-p$ -

\section{Introduction}

On the strength of the recent report of atmospheric neutrino oscillations [1], as well as previous other indications of solar [2ind and accelerator [3] neutrino oscillations, neutrino masses are now considered to be almost established experimentally. Yet there is no clear theoretical consensus as to the origin of neutrino masses. In the standard model, the usual way is to add three right-handed neutrino singlets with large Majorana masses and use the canonical seesaw mechanism [inin to obtain small Majorana masses for $\nu_{e}, \nu_{\mu}$, and $\nu_{\tau}$. On the other hand, other mechanisms are available [in $[\overline{1}]$, the simplest alternative being the addition of a heavy scalar triplet [i, $\left.\overline{6}_{i}^{\overline{6}}\right]$.

There is another important theoretical reason for going beyond the minimal standard model, i.e. supersymmetry. However, the minimal supersymmetric standard model (MSSM) keeps the neutrinos massless because it contains no extra fields or interactions which could make them massive. Of course, one may simply add [i];] three right-handed neutrino singlet superfields to the MSSM and invoke the canonical seesaw mechanism as before. On the other hand, given the particle content of the MSSM, one may also allow new, lepton-number nonconserving terms in the Lagrangian which would then induce nonzero

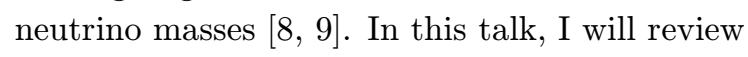
briefly this latter situation where $R$-parity is usually assumed to be violated, and point out its potential problem with leptogenesis, ending with a proposal of radiative neutrino masses with $R$ - parity conservation.

\section{MSSM and R-Parity}

The well-known superfield content of the MSSM is given by

$$
\begin{aligned}
& Q_{i}=\left(u_{i}, d_{i}\right)_{L} \sim(3,2,1 / 6), \\
& u_{i}^{c} \sim\left(3^{*}, 1,-2 / 3\right), \\
& d_{i}^{c} \sim\left(3^{*}, 1,1 / 3\right), \\
& L_{i}=\left(\nu_{i}, l_{i}\right)_{L} \sim(1,2,-1 / 2), \\
& l_{i}^{c} \sim(1,1,1) ; \\
& H_{1}=\left(\bar{\phi}_{1}^{0},-\phi_{1}^{-}\right) \sim(1,2,-1 / 2), \\
& H_{2}=\left(\phi_{2}^{+}, \phi_{2}^{0}\right) \sim(1,2,1 / 2) .
\end{aligned}
$$

Given the above transformations under the standard $S U(3) \times S U(2) \times U(1)$ gauge group, the corresponding superpotential should contain in general all gauge-invariant bilinear and trilinear combinations of the superfields. However, to forbid the nonconservation of both baryon number $(B)$ and lepton number $(L)$, each particle is usually assigned a dicrete $R$-parity:

$$
R \equiv(-1)^{3 B+L+2 j},
$$

which is assumed to be conserved by the allowed interactions. Hence the MSSM superpotential has only the terms $H_{1} H_{2}, H_{1} L_{i} l_{j}^{c}, H_{1} Q_{i} d_{j}^{c}$, and $H_{2} Q_{i} u_{j}^{c}$. Since the superfield $\nu_{i}^{c} \sim(1,1,0)$ is absent, $m_{\nu}=0$ in the MSSM as in the minimal

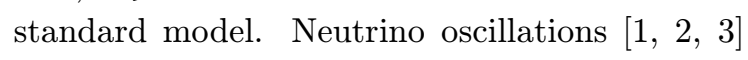
are thus unexplained. 
Phenomenologically, it makes sense to require only $B$ conservation (to make sure that the proton is stable), but to allow $L$ violation (hence $R$-parity violation) so that the additional terms $L_{i} H_{2}, L_{i} L_{j} l_{k}^{c}$, and $L_{i} Q_{j} d_{k}^{c}$ may occur. Note that they all have $\Delta L=1$. ¿From the bilinear terms [9.0.

$$
-\mu H_{1} H_{2}+\epsilon_{i} L_{i} H_{2},
$$

we get a $7 \times 7$ neutralino-neutrino mass matrix

$$
\left[\begin{array}{ccccc}
M_{1} & 0 & -g_{1} v_{1} & -g_{1} v_{2} & -g_{1} u_{i} \\
0 & M_{2} & g_{2} v_{1} & -g_{2} v_{2} & g_{2} u_{i} \\
-g_{1} v_{1} & g_{2} v_{1} & 0 & -\mu & 0 \\
g_{1} v_{2} & -g_{2} v_{2} & -\mu & 0 & \epsilon_{i} \\
-g_{1} u_{i} & g_{2} u_{i} & 0 & \epsilon_{i} & 0
\end{array}\right]
$$

where $v_{1,2}=\left\langle\phi_{1,2}^{0}\right\rangle / 2$ and $u_{i}=\left\langle\tilde{\nu}_{i}\right\rangle / 2$, with $i=e, \mu, \tau$. Note first the important fact that a nonzero $\epsilon_{i}$ implies a nonzero $u_{i}$ [ịin]. Note also that if $u_{i} / \epsilon_{i}$ is the same for all $i$, then only one linear combination of the three neutrinos gets a tree-level mass. From the trilinear terms, neu-

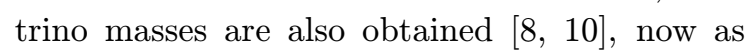
one-loop radiative corrections. Note that these occur as the result of supersymmetry breaking and are suppressed by $m_{d}^{2}$ or $m_{l}^{2}$.

\section{3. $L$ Nonconservation and the Uni- verse}

As noted earlier, the $R$-parity nonconserving interactions have $\Delta L=1$. Furthermore, the particles involved have masses at most equal to the supersymmetry breaking scale, i.e. a few TeV. This means that their $L$ violation together with the $B+L$ violation by sphalerons [1] 1, ] would erase any primordial $B$ or $L$ asymmetry of the Universe $\left[1 \overline{2}_{1}^{1}, 1133_{1}^{1}\right.$. To avoid such a possibility, one may reduce the relevant Yukawa couplings to less than about $10^{-7}$, but a typical minimum value of $10^{-4}$ is required for realistic neutrino masses. Hence the existence of the present baryon asymmetry of the Universe is unexplained if neutrino masses originate from these $\Delta L=1$ interactions. This is a generic problem of all models of radiative neutrino masses where the $L$ violation can be traced to interactions occuring at energies below $10^{13}$ $\mathrm{GeV}$ or so.
Consider the prototype (Zee) model of radiative neutrino masses [i ric and it only adds one charged scalar singlet $\chi^{ \pm}$ and a second Higgs doublet to the minimal standard model. Call the two Higgs doublets $\Phi_{1,2}$, then the trilinear coupling $\chi^{-}\left(\phi_{1}^{+} \phi_{2}^{0}-\phi_{1}^{0} \phi_{2}^{+}\right)$is allowed as well as the Yukawa coupling $\chi^{+}\left(\nu_{i} l_{j}-\right.$ $\left.l_{i} \nu_{j}\right)$. Hence there is an effective dimension-5 operator $\nu_{i} \nu_{j} \phi_{1}^{0} \phi_{2}^{0}$ which renders the neutrinos massive [i5in, but it is again suppressed by $m_{l}^{2}$. Note that the new interactions have $\Delta L=2$.

\section{Supersymmetric Radiative Neu- trino Masses and Leptogenesis}

It has been shown recently [i6] that naturally small Majorana neutrino masses may be obtained from heavy scalar triplets and if the latter have masses of order $10^{13} \mathrm{GeV}$, their decays could generate a lepton asymmetry which then gets converted into the present baryon asymmetry of the Universe through the electroweak phase transition. The same role may be attributed to the scalar singlets of the Zee model if they are heavy enough, but then to obtain realistic radiative neutrino masses, unsuppressed Yukawa couplings are needed.

Consider now the following supersymmetric extension of the Zee model. Since all the interactions are either $\Delta L=0$ or $\Delta L=2, R$-parity is conserved. Because of the requirement of supersymmetry, there is a doubling of the scalar superfields:

$$
\begin{aligned}
& \chi_{1}^{+} \sim(1,1,1 ;+,-), \\
& \chi_{2}^{-} \sim(1,1,-1 ;+,-), \\
& H_{1,3} \sim(1,2,-1 / 2 ;+, \pm), \\
& H_{2,4} \sim(1,2,1 / 2 ;+, \pm) .
\end{aligned}
$$

A fourth family of leptons is then added:

$$
\begin{aligned}
& \left(N_{1}^{0}, E^{-}\right) \sim(1,2,-1 / 2 ;-,-), \\
& N_{2}^{0} \sim(1,1,0 ;-,-), \\
& E^{+} \sim(1,1,1 ;-,-) .
\end{aligned}
$$

In the above, the assignments of these superfields under a discrete $Z_{2} \times Z_{2}^{\prime}$ symmetry are also displayed. The first is merely the one usually assumed to obtain $R$-parity; the second is used to distinguish the new particles from those of 
the MSSM. The relevant terms in the $R$-parity preserving superpotential of this model are then given by

$$
\begin{aligned}
W & =\mu_{12}\left(h_{1}^{0} h_{2}^{0}-h_{1}^{-} h_{2}^{+}\right) \\
& +\mu_{34}\left(h_{3}^{0} h_{4}^{0}-h_{3}^{-} h_{4}^{+}\right) \\
& +m_{\chi} \chi^{+} \chi^{-} \\
& +\left(m_{E} / v_{1}\right)\left(h_{1}^{0} E^{-}-h_{1}^{-} N_{1}^{0}\right) E^{+} \\
& +f_{i}\left(\nu_{i} h_{3}^{-}-l_{i} h_{3}^{0}\right) E^{+} \\
& +f_{j}^{\prime}\left(\nu_{j} E^{-}-l_{j} N_{1}^{0}\right) \chi_{1}^{+} \\
& +f_{24}\left(h_{2}^{+} h_{4}^{0}-h_{2}^{0} h_{4}^{+}\right) \chi_{2}^{-},
\end{aligned}
$$

where $v_{1,2}$ are the vacuum expectation values of $h_{1,2}^{0}$. The unsuppressed one-loop diagram generating neutrino masses is shown in Fig. 1 of Ref. [13]. Note that the effective supersymmetric dimension-5 operator $L_{i} L_{j} H_{2} H_{2}$ is indeed realized. Assuming that the masses of the scalar leptons of the fourth family to be equal to $M_{S U S Y}$, the neutrino mass matrix is then obtained:

$$
\frac{\left(f_{i} f_{j}^{\prime}+f_{i}^{\prime} f_{j}\right) f_{24} v_{2}^{2} m_{E} \mu_{12} \mu_{34}}{16 \pi^{2} v_{1} M_{S U S Y}^{2} m_{\chi}} \ln \frac{m_{\chi}^{2}}{M_{S U S Y}^{2}} .
$$

To get an estimate of the above expression, let $f_{i}=f_{j}^{\prime}=f_{24}=1, m_{E}=v_{1}, \mu_{12}=\mu_{34}=$ $M_{S U S Y}$, then

$$
m_{\nu}=\frac{1}{8 \pi^{2}} \frac{v_{2}^{2}}{m_{\chi}} \ln \frac{m_{\chi}^{2}}{M_{S U S Y}^{2}} .
$$

Assuming $v_{2} \sim 10^{2} \mathrm{GeV}, m_{\chi} \sim 10^{13} \mathrm{GeV}$, and $M_{S U S Y} \sim 10^{3} \mathrm{GeV}$, a value of $m_{\nu} \sim 0.6 \mathrm{eV}$ is obtained. This is just one order of magnitude greater than the square root of the $\Delta m^{2} \sim$ $5 \times 10^{-3} \mathrm{eV}^{2}$ needed for atmospheric neutrino oscillations [i]. Reducing slightly the above dimensionless couplings from unity would fit the data quite well. Since $m_{\chi} \sim 10^{13} \mathrm{GeV}$ is now allowed, leptogenesis should be possible as demonstrated in Ref. [6].

\section{Neutrino Oscillations}

It has recently been shown [155, that the structure of Eq. (4.9) for the $\mu-\tau$ sector is naturally suited for the large mixing solution of atmospheric neutrino oscillations. To be more specific, the $2 \times 2$ submatrix of Eq. (4.9) for the $\mu-\tau$ sector can be written as

$$
m_{0}\left[\begin{array}{cc}
2 \sin \alpha \sin \alpha^{\prime} & \sin \left(\alpha+\alpha^{\prime}\right) \\
\sin \left(\alpha+\alpha^{\prime}\right) & 2 \cos \alpha \cos \alpha^{\prime}
\end{array}\right]
$$

where $\tan \alpha=f_{\mu} / f_{\tau}$ and $\tan \alpha^{\prime}=f_{\mu}^{\prime} / f_{\tau}^{\prime}$. The eigenvalues of the above are then given by $m_{0}\left(c_{1} \pm\right.$ 1 ), where $c_{1}=\cos \left(\alpha-\alpha^{\prime}\right)$, and the effective $\sin ^{2} 2 \theta$ for $\nu_{\mu}-\nu_{\tau}$ oscillations is $\left(1-c_{2}\right) /\left(1+c_{2}\right)$, where $c_{2}=\cos \left(\alpha+\alpha^{\prime}\right)$. If $\tan \alpha \sim \tan \alpha^{\prime} \sim 1$, then $c_{1} \sim 1$ and $c_{2} \sim 0$. In that case, maximal mixing between a heavy $\left(2 m_{0}\right)$ and a light $\left(s_{1}^{2} m_{0} / 2\right)$ neutrino occurs as an explanation of the atmospheric data. If it is assumed further that $f_{e}<<f_{\mu, \tau}$ and $f_{e}^{\prime}<<f_{\mu, \tau}^{\prime}$, then the smallangle matter-enhanced solution of solar neutrino oscillations may be obtained as well.

\section{Collider Phenomenology}

The above model has the twin virtues of an acceptable neutrino mass matrix and the possibility of generating a lepton asymmetry of the Universe. It is also phenomenologically safe because all the additions to the standard model do not alter its known successes. Neither the fourth family of leptons nor the two extra Higgs doublets mix with their standard-model analogs because they are odd under the new discrete $Z_{2}^{\prime}$ symmetry. In particular, $H_{3}$ and $H_{4}$ do not couple to the known quarks and leptons, hence flavorchanging neutral currents are suppressed here as in the standard model. The lepton-number violation of this model is associated with $m_{\chi}$ which is of order $10^{13} \mathrm{GeV}$. However, the fourth family of leptons should have masses of order $10^{2}$ $\mathrm{GeV}$ and be observable at planned future colliders. The two extra Higgs doublets should also be observable with an energy scale of order $M_{S U S Y}$. The soft supersymmetry-breaking terms of this model are assumed to break $Z_{2}^{\prime}$ without breaking $Z_{2}$. Hence there will still be a stable LSP (lightest supersymmetric particle) and a fourth-family lepton will still decay into ordinary leptons. For example, because $\tilde{h}_{3}^{0}$ mixes with $\tilde{h}_{1}^{0}$, the decay

$$
E^{-} \rightarrow \mu^{-} \tilde{h}_{3}^{0}\left(\tilde{h}_{1}^{0}\right) \rightarrow \mu^{-} \tau^{+} \tau^{-}
$$

is possible and would make a spectacular signature. 


\section{Conclusion}

In conclusion, the issue of neutrino masses in supersymmetry has been addressed in this talk. The assumption of $R$-parity nonconservation is shown to be generically inconsistent with leptogenesis because the lepton-number violating interactions would act in conjunction with the $B+$ $L$ violating sphaleron processes and erase any pre-existing $B$ or $L$ or $B-L$ asymmetry of the Universe. This constraint means that any $R$ parity violation must be very small, so that it is of negligible phenomenological interest and cannot contribute significantly to neutrino masses. This conclusion also applies to models of radiative neutrino masses with suppressed Yukawa couplings, such as the Zee model. However, it has also been shown that realistic neutrino masses in supersymmetry are possible beyond the MSSM with $R$-parity conservation where lepton-number violation is by two units and occurs at the mass scale of $10^{13} \mathrm{GeV}$. The specific model presented also predicts new particles which should be observable in the future at the LHC (Large Hadron Collider).

\section{Acknowledgments}

I thank the organizers George Zoupanos, Nick Tracas, and George Koutsoumbas for their great hospitality at Corfu. This work was supported in part by the U. S. Department of Energy under Grant No. DE-FG03-94ER40837.

\section{References}

[1] Y. Fukuda et al., Phys. Lett. B433, 9 (1998); B436, 33 (1998); Phys. Rev. Lett. 81, 1562 (1998).

[2] R. Davis, Prog. Part. Nucl. Phys. 32, 13 (1994); Y. Fukuda et al., Phys. Rev. Lett. 77, 1683 (1996); 81, 1158 (1998); P. Anselmann et al., Phys. Lett. B357, 237 (1995); B361, 235 (1996); J. N. Abdurashitov et al., Phys. Lett. B328, 234 (1994).

[3] C. Athanassopoulos et al., Phys. Rev. Lett. 75, 2650 (1995); 77, 3082 (1996); 81, 1774 (1998).
[4] M. Gell-Mann, P. Ramond, and R. Slansky, in Supergravity, edited by P. van Nieuwenhuizen and D. Z. Freedman (North-Holland, Amsterdam, 1979), p. 315; T. Yanagida, in Proceedings of the Workshop on the Unified Theory and the Baryon Number in the Universe, edited by $\mathrm{O}$. Sawada and A. Sugamoto (KEK Report No. 7918, Tsukuba, Japan, 1979), p. 95; R. N. Mohapatra and G. Senjanovic, Phys. Rev. Lett. 44, 1316 (1980).

[5] E. Ma, Phys. Rev. Lett. 81, 1171 (1998).

[6] E. Ma and U. Sarkar, Phys. Rev. Lett. 80, 5716 (1998).

[7] See for example Q. Shafi and Z. Tavartkiladze, hep-ph/9811463.

[8] L. Hall and M. Suzuki, Nucl. Phys. B231, 419 (1984); H.-P. Nilles and N. Polonsky, Nucl. Phys. B484, 33 (1997)..

[9] M. A. Diaz, J. C. Romao, and J. W. F. Valle, Nucl. Phys. B524, 23 (1998).

[10] M. Drees, S. Pakvasa, X. Tata, and T. ter Veldhuis, Phys. Rev. D57, R5340 (1998).

[11] V. A. Kuzmin, V. A. Rubakov, and M. E. Shaposhnikov, Phys. Lett. 155B, 36 (1985).

[12] B. A. Campbell, S. Davidson, J. E. Ellis, and K. Olive, Phys. Lett. B256, 457 (1991).

[13] E. Ma, M. Raidal, and U. Sarkar, hep$\mathrm{ph} / 9901406$.

[14] A. Zee, Phys. Lett. 93B, 389 (1980).

[15] E. Ma, Phys. Lett. B442, 238 (1998). 